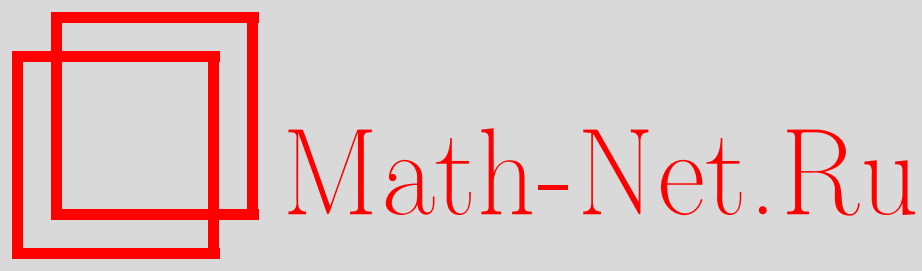

А. Д. Миронов, А. Ю. Морозов, Излучение вне рамок четырех измерений, ТMФ, 2008, том 156, номер 2, 282 291

DOI: https://doi.org/10.4213/tmf6247

Использование Общероссийского математического портала Math-Net.Ru подразумевает, что вы прочитали и согласны с пользовательским соглашением http://www . mathnet.ru/rus/agreement

Параметры загрузки:

IP : 54.81 .137 .203

26 апреля 2023 г., 12:37:58

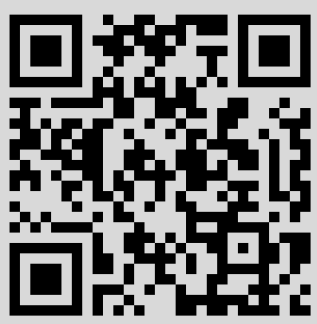




\section{ИЗЛУЧЕНИЕ ВНЕ РАМОК ЧЕТЫРЕХ ИЗМЕРЕНИЙ}

Представлен набор формул, описывающих классическое излучение тензорного поля ранга $s$ ускоренно движущимся точечным источником в плоском пространстве-времени произвольной четной размерности $d$. Эти формулы позволяют непосредственно и алгоритмически вычислить полную интенсивность и излученный импульс для любых $s$ и $d$ на бумаге или при помощи компьютера. Практическое применение полученных результатов для $s>1$ ограниченно из-за несохранения тензора энергии-импульса точечной частицы. Это обычно означает, что нельзя пренебрегать вкладами в излучение от напряжений сил, которые приводят к ускорению излучающего источника.

Ключевые слова: классическое излучение, старшие измерения, теория струн.

Традиционно процессы излучения рассматривались как предмет непосредственных физических приложений и, следовательно, глубоко исследовались только в пространстве-времени размерности не большей 4 [1]. Даже в размерности $d=2$ и $d=3$, где существуют очевидные применения, скажем, к звуковым волнам в среде, теория плохо представлена в литературе. И лишь недавно, после того как многомерные модели, инспирированные теорией струн [2], привлекли растущий интерес [3], начали появляться некоторые статьи, посвященные многомерному излучению [4]-[8]. Их, конечно, все еще слишком мало для того, чтобы исчерпать предмет на том уровне подробности, который характерен для литературы, посвященной излучению в четырех измерениях. В данной статье мы делаем шаг, который, как нам кажется, необходим для систематического изучения физических эффектов. Мы выводим общие формулы, описывающие классическое излучение в произвольной размерности $d$ и для произвольного ранга $s$ поля излучения. Это должно помочь прояснить физические и математические структуры, которые стоят за излучением в высших измерениях. В частности, используя наши результаты, можно непосредственно рассчитать силу радиационного трения в высших измерениях, к примеру методом, описанным в статье [4].

Чтобы найти интенсивность излучения, нужно выполнить следующую цепочку вычислений.

${ }^{*}$ Институт теоретической и экспериментальной физики, Москва, Россия.

E-mail: mironov@itep.ru,morozov@itep.ru

${ }^{\dagger}$ Физический институт им. П. Н. Лебедева РАН, Москва, Россия. E-mail: mironov@lpi.ru 
ШАГ 1. Решить волновое уравнение для точечного источника поля ранга $s$, движущегося вдоль мировой линии $z^{\mu}(\tau)$,

$$
\square A_{\mu_{1} \ldots \mu_{s}}(x)=\oint u_{\mu_{1}} \ldots u_{\mu_{s}} \delta^{(d)}(x-z(\tau)) d \tau,
$$

и выделить вклад, спадающий медленнее всего на больших расстояниях. Для четных $d$ он дается простой формулой для запаздывающего потенциала Лиенара-Вихерта:

$$
\begin{gathered}
A_{\mu_{1} \ldots \mu_{s}}^{\mathrm{rad}}=\left(\frac{1}{(R u)} \partial_{\tau}\right)^{\frac{d-4}{2}} \frac{u_{\mu_{1}} \ldots u_{\mu_{s}}}{R u}, \\
\partial_{\mu} A_{\mu_{1} \ldots \mu_{s}}^{\mathrm{rad}}=R_{\mu}\left(\frac{1}{(R u)} \partial_{\tau}\right)^{\frac{d-2}{2}} \frac{u_{\mu_{1}} \ldots u_{\mu_{s}}}{R u},
\end{gathered}
$$

где $u_{\mu}=\partial_{\tau} z_{\mu}$ есть $d$-скорость источника $\left(u^{2}=1\right)$, вычисленная в момент излучения $t^{\prime}=z^{0}(\tau)$, определенный условием $R^{2}=0$, где $R_{\mu}-d$-вектор с компонентами $R^{\mu} \equiv$ $x^{\mu}-z^{\mu}(\tau)$. Здесь $\tau-$ собственное время источника. В частности,

$$
\frac{\partial \tau}{\partial x^{\mu}}=\frac{R_{\mu}}{(R u)} .
$$

Мы также вводим обозначение $n^{\mu} \equiv(1,-\vec{n})=R^{\mu} / R$, где $R$ - длина пространственной части $R^{\mu}, R \equiv \sqrt{\left(R^{i}\right)^{2}} \quad\left(n^{\mu}\right.$ не является $d$-вектором в противоположность $R^{\mu}$; мы определяем его в лабораторной системе).

Удобно переписать (2) в сжатых обозначениях

$$
\begin{aligned}
A_{\mathrm{rad}} & =\frac{1}{R^{d-2}}\left(\frac{1}{U} \partial_{\tau}\right)^{p-1} \frac{S}{U}=\frac{1}{R^{d-2}} \sum_{q=0}^{p-1}\left(\partial_{\tau}^{q} S\right) A_{q}^{p-1}, \\
\partial_{\mu} A_{\mathrm{rad}} & =\frac{n_{\mu}}{R^{d-2}} \sum_{q=0}^{p}\left(\partial_{\tau}^{q} S\right) A_{q}^{p}
\end{aligned}
$$

где $U=(n u), S=u_{\mu_{1}} \ldots u_{\mu_{s}}, p=(d-2) / 2$, и находить $A_{q}^{p}$, пользуясь рекуррентными соотношениями

$$
A_{q}^{p+1}=\frac{1}{U}\left(\partial_{\tau} A_{q}^{p}+A_{q-1}^{p}\right) .
$$

ШАГ 2. Проверить поперечность. В лидирующем порядке по $1 / R$, когда можно пренебречь производными по $R$,

$$
\partial^{\mu_{s}} A_{\mu_{1} \mu_{2} \ldots \mu_{s}}^{\mathrm{rad}}=\left(\frac{1}{(R u)} \partial_{\tau}\right)^{\frac{d-2}{2}} u_{\mu_{1}} \ldots u_{\mu_{s-1}}
$$

не исчезает при $s \geqslant 2$, и это нельзя исправить вычитанием следов. Физическая причина, приводящая к непоперечности, состоит в том, что мы пренебрегаем излучением от напряжений сил, которые приводят к ускорению источника: только учет всех напряжений делает проблему излучения хорошо определенной для $s \geqslant 2$. Формулы данной статьи для $s \geqslant 2$ дают только часть полного ответа. 
ШАГ 3. Построить тензор энергии-импульса в волновой зоне. Он равен

$$
T_{\mu \nu}^{(s)}=R_{\mu} R_{\nu}\left\{\left(\frac{1}{(R u)} \partial_{\tau}\right)^{\frac{d-2}{2}} \frac{u_{\mu_{1}} \ldots u_{\mu_{s}}}{R u}\right\}^{2}=\frac{R_{\mu} R_{\nu}}{R^{d}}\left\{\sum_{q=0}^{p} A_{q}^{p} \partial_{\tau}^{q} S\right\}^{2} .
$$

В приложениях возникают различные линейные комбинации таких тензоров энергии-импульса. К примеру, для скалярных волн

$$
T_{\mu \nu}^{(\text {scalar })}=-T_{\mu \nu}^{(0)}
$$

Причина возникновения знака минус состоит в том, что для всех полей ненулевого спина только пространственные компоненты имеют физический смысл (т.е. сохраняются в физических калибровках), что приводит к общему знаку минус в кинетической части тензора энергии-импульса по сравнению со скалярным полем. Для гравитационных волн

$$
T_{\mu \nu}^{(\text {grav })}=T_{\mu \nu}^{(2)}-\frac{1}{2} T_{\mu \nu}^{(0)}
$$

Подобное переопределение необходимо также для высших спинов $s>2$. Результаты для таких линейно преобразованных величин могут быть легко получены с использованием формул для $T_{\mu \nu}^{(s)}$.

ШАГ 4. Вычислить поток излучаемого импульса через сферу радиуса $R$. Он равен

$$
d \mathcal{P}_{d \mid s}^{\mu}=-\oint\left\{T^{\mu i} n_{i} d x^{0}\right\} d S
$$

где $d S$ - инфинитезимальный элемент этой сферы (т.е. интегрирование ведется по сфере). Таким образом, окончательно получаем (используя равенство $d x^{0}=U d \tau=$ $(n u) d \tau)$

$$
\begin{aligned}
\frac{\partial \mathcal{P}_{d \mid s}^{\mu}}{d \tau} & =-\oint T^{\mu i} n_{i} R^{d-2} U d \Omega_{d-2}= \\
& =\sum_{q^{\prime}, q^{\prime \prime}=0}^{p}\left(\partial_{\tau}^{q^{\prime}}\left(u_{\mu_{1}} \ldots u_{\mu_{s}}\right) \partial_{\tau}^{q^{\prime \prime}}\left(u^{\mu_{1}} \ldots u^{\mu_{s}}\right)\right) \int n^{\mu} U A_{q^{\prime}}^{p} A_{q^{\prime \prime}}^{p} d \Omega_{d-2}(n)= \\
& =\sum_{k=0}^{\frac{d-4}{2}}\left(\partial_{\tau}^{k} u^{\mu}\right) P_{k}^{(d \mid s)}(\kappa) .
\end{aligned}
$$

где $d \Omega_{d-2}$ - телесный угол в $(d-1)$-мерном пространстве, а $P_{k}^{(d \mid s)}$ - функции различных скалярных произведений $\left(\partial_{\tau}^{l} u_{\nu} \partial_{\tau}^{m} u^{\nu}\right)$ с $l+m \leqslant d-4-k$. В частности, потери энергии на излучение равны $\left(d t^{\prime}=u^{0} d \tau=\gamma d \tau\right)$

$$
\frac{d \mathcal{P}_{d \mid s}^{0}}{d t^{\prime}}=\frac{1}{\gamma} \sum_{k=0}^{\frac{d-4}{2}}\left(\partial_{\tau}^{k} \gamma\right) P_{k}^{(d \mid s)}(\kappa) \stackrel{\gamma=\left(1-v^{2}\right)^{-1 / 2}=\mathrm{const}}{\longrightarrow} P_{0}^{(d \mid s)}(\kappa) .
$$


Снова подчеркнем, что эта величина дает только часть полного ответа для $s \geqslant 2$ из-за эффектов, подобных тем, которые описываются формулами (6) и (8), (9). Более того, она может быть даже отрицательной (!): к примеру, в случае асимптотически большого ранга $s$ лидирующий вклад в излученный импульс $\frac{d \mathcal{P}_{d \mid s}^{\mu}}{d \tau}$ ведет себя как $-\left(s \dot{u}^{2}\right)^{d / 2-1} \sim(-1)^{d / 2}$ с учетом того, что $\dot{u}^{2} \leqslant 0$. Для $s=0$ отрицательность исправляется изменением общего знака (см. (8)), но для $s \geqslant 3$ процедура должна быть более хитрой.

Иногда рассматривают также свертки $I_{d \mid s}=u^{\mu} \frac{d \mathcal{P}_{d \mid s}^{\mu}}{d \tau}[6]$, однако они не имеют прямого физического смысла, если только не верно, что $\frac{d \mathcal{P}_{d \mid s}^{\mu}}{d \tau} \sim u_{\mu}$, как это случается для $d=4$. Только тогда $I_{d \mid s}=\frac{d \mathcal{P}_{d \mid s}^{0}}{d t^{\prime}}$.

ШАГ 5. Вычислить угловые интегралы по изотропным (светоподобным) единичным векторам $n^{\mu}$ в (11) (хотя $n^{\mu}$ не является $d$-вектором, эти интегралы лоренц-инвариантны, поскольку выражение (11) лоренц-инвариантно),

$$
\int \frac{n^{\mu_{1}} \ldots n^{\mu_{m}}}{(n u)^{d+m-2}} d \Omega_{d-2}(n) \sim \operatorname{Pr}_{d}\left(u^{\mu_{1}} \ldots u^{\mu_{m}}\right) .
$$

Интегралы выражаются через проектор на бесследовые состояния $\operatorname{Pr}_{d}$ (поскольку $n^{2}=0$ и свертка любой пары $\mu$-индексов в левой части (13) должна давать нуль):

$$
\begin{array}{cc}
\operatorname{spin} s=2: & \operatorname{Pr}_{d}\left(u_{\mu} u_{\nu}\right)=u_{\mu} u_{\nu}-\frac{1}{d} \eta_{\mu \nu} ; \\
\operatorname{spin} s=3: & \operatorname{Pr}_{d}\left(u_{\mu_{1}} u_{\mu_{2}} u_{\mu_{3}}\right)=u_{\mu_{1}} u_{\mu_{2}} u_{\mu_{3}}- \\
& -\frac{1}{d+2}\left(\eta_{\mu_{1} \mu_{2}} u_{\mu_{3}}+\eta_{\mu_{1} \mu_{3}} u_{\mu_{2}}+\eta_{\mu_{2} \mu_{3}} u_{\mu_{1}}\right) ;
\end{array}
$$

spin $s=4: \quad \operatorname{Pr}_{d}\left(u_{\mu_{1}} u_{\mu_{2}} u_{\mu_{3}} u_{\mu_{4}}\right)=u_{\mu_{1}} u_{\mu_{2}} u_{\mu_{3}} u_{\mu_{4}}-$

$$
\begin{aligned}
& -\frac{1}{d+4}\left(\eta_{\mu_{1} \mu_{2}} u_{\mu_{3}} u_{\mu_{4}}+5 \text { перестановок }\right)+ \\
& +\frac{1}{(d+4)(d+2)}\left(\eta_{\mu_{1} \mu_{2}} \eta_{\mu_{3} \mu_{4}}+\eta_{\mu_{1} \mu_{3}} \eta_{\mu_{2} \mu_{4}}+\eta_{\mu_{1} \mu_{4}} \eta_{\mu_{2} \mu_{3}}\right)
\end{aligned}
$$

Для произвольного $m$ мы имеем (здесь $[m / 2]$ обозначает целую часть числа $m / 2$ )

$$
\begin{aligned}
& \operatorname{Pr}_{d}\left(u_{\mu_{1}} \ldots u_{\mu_{m}}\right)=\sum_{k=0}^{[m / 2]}(-1)^{k} \frac{(d+2 m-4-2 k) ! !}{(d+2 m-4) ! !} \times \\
& \times(\overbrace{u_{\mu_{1}} \ldots u_{\mu_{m-2 k}}(\underbrace{\eta_{\mu_{m-2 k+1} \mu_{m-2 k+2} \ldots \eta_{\mu_{m-1} \mu_{m}}+\cdots}+\cdots}_{(2 k-1) ! !})+\cdots}^{\text {перестановок }}) .
\end{aligned}
$$

Этот проектор удобно записать при помощи производящей функции: после свертки с $m$ копиями $d$-вектора $x$ мы получим

$$
\operatorname{Pr}_{d}\left((x u)^{m}\right)=\sum_{k=0}^{[m / 2]}(-1)^{k}(2 k-1) ! ! C_{2 k}^{m} \frac{(d+2 m-4-2 k) ! !}{(d+2 m-4) ! !}(x u)^{m-2 k}\left(x^{2}\right)^{k}=
$$




$$
=\sum_{k=0}^{[m / 2]}(-1)^{k} \frac{(d+2 m-4-2 k) ! !}{(d+2 m-4) ! !} \frac{m !(x u)^{m-2 k}\left(x^{2}\right)^{k}}{2^{k} k !(m-2 k) !} .
$$

Чтобы определить нормировочный множитель в (13), нужно знать сумму коэффициентов ряда (14),

$$
\begin{aligned}
c_{d}(m) & =\sum_{k=0}^{[m / 2]}(-1)^{k} C_{2 k}^{m}(2 k-1) ! ! \frac{(d+2 m-4-2 k) ! !}{(d+2 m-4) ! !}= \\
& =\frac{(d+2[m / 2]-3) ! !(d+2(m-[m / 2])-4) ! !}{(d-3) ! !(d+2 m-4) ! !} .
\end{aligned}
$$

Переписанные в терминах производящей функции с восстановленным нормировочным множителем, угловые интегралы (13) имеют вид

$$
\begin{aligned}
& \int \frac{(n x)^{m} d \Omega_{d-2}(n)}{(n u)^{d+m-2}}=\frac{S_{n-2}}{c_{d}(s)} \operatorname{Pr}_{d}\left((u x)^{m}\right)=\frac{2^{\frac{d}{2}} \pi^{\frac{d-2}{2}}}{(d+2[m / 2]-3) ! !} \times \\
& \quad \times \sum_{j=0}^{[m / 2]}(-1)^{j} \frac{(d+2 m-4-2 j) ! !}{(d+2 m-2[m / 2]-4) ! !} \frac{m !}{2^{j} j !(m-2 j) !}(u x)^{m-2 j}\left(x^{2}\right)^{j} .
\end{aligned}
$$

Остается подставить вместо вектора $x$ вектор $t x_{0}+\sum_{k=0}^{\frac{d-2}{2}} a_{k} \partial_{\tau}^{k} u$ и выделить коэффициент перед подходящей комбинацией $t$ и $a_{k}$. Заметим, что в этом вычислении используется производящая функция, что приводит к дополнительному комбинаторному коэффициенту, который необходимо явно учесть. Например, для того чтобы получить правильный коэффициент перед членом $\partial_{\tau}^{k_{1}} u \ldots \partial_{\tau}^{k_{n}} u$ с различающимися значениями $k_{1}, \ldots, k_{n}$, нужно разделить производящую функцию на $n$ !.

Окончательный результат может быть выражен как через скалярные произведения $U_{i j} \equiv \partial_{\tau}^{l} \vec{u}_{\nu} \partial_{\tau}^{m} \vec{u}^{\nu}$, так и через кривизны Френе $\kappa_{m}$ и их производные $\partial_{\tau}^{l} \kappa_{m}$. Таким образом, последний шаг состоит в следующем.

ШАГ 6. Выразить $U_{i j}$ через кривизны Френе [9], параметризующие сопутствующий ортонормированный базис, ассоциированный с мировой линией $x^{\mu}(\tau)$. Он формируется из $d$-векторов $\vec{N}^{(\mu)}, \mu=0, \ldots, d-1$,

$$
\vec{N}^{(\mu)} \vec{N}^{(\nu)}=\eta^{\mu \nu}
$$

с $\vec{N}^{(0)}=\vec{u}\left(\right.$ т.е. $\left.N_{\mu}^{(0)}=u_{\mu}\right)$, и остальных векторов, заданных рекуррентным соотношением

$$
\partial_{\tau} \vec{N}^{(\mu)}=-k_{\mu+1} \vec{N}^{(\mu+1)}+\sum_{\nu=1}^{\mu} \beta_{\mu \nu} \vec{N}^{(\nu)} .
$$

Дифференцируя по $\tau$ условие ортонормированности, мы получим

$$
\left(\partial_{\tau} \vec{N}^{(\mu)}\right) \vec{N}^{(\nu)}+\vec{N}^{(\mu)}\left(\partial_{\tau} \vec{N}^{(\nu)}\right)=0 .
$$

Отсюда следует, что при $\nu \leqslant \mu$

$$
\beta_{\mu \nu}=\left(\partial_{\tau} \vec{N}^{(\mu)}\right) \vec{N}^{(\nu)}=-\vec{N}^{(\mu)}\left(\partial_{\tau} \vec{N}^{(\nu)}\right)=\kappa_{\mu} \delta_{\nu, \mu-1} \eta^{\mu \mu}
$$


И

$$
\partial_{\tau} \vec{N}^{(\mu)}=-k_{\mu+1} \vec{N}^{(\mu+1)}+k_{\mu} \vec{N}^{\mu-1} .
$$

Параметры $k_{\mu}$ (это - не $d$-векторы!) зависят от формы мировой линии $x^{\mu}(\tau)$ в инфинитезимальной окрестности точки и называются кривизнами Френе.

Подведем итог. Все указанные шаги легко делаются с помощью программ MAPLE или Mathematica ${ }^{1)}$. В приложении мы получаем явные формулы для излученного $d$-импульса $d \mathcal{P}_{d \mid s}^{\mu}$, а также несколько более простые формулы для $P_{0}^{(d \mid s)}$ - интенсивности излучения при постоянном $\gamma\left(\kappa_{2}^{2}=\frac{\gamma^{2} \kappa_{1}^{2}}{\gamma^{2}-1}=\right.$ const, $\left.\kappa_{i}=0, i>2\right)$, см. (12), и для сверток $I_{d \mid s}=u^{\mu} \frac{d \mathcal{P}_{d \mid s}^{\mu}}{d \tau}[6]$ при "реалистичных" значениях $d=4,6,8,10$ и произвольном ранге $s$. Сначала мы приводим результаты для случаев $d=4,6,8$, для которых формулы относительно просты, и можно понять их общую структуру. Значительно более запутанные формулы в наиболее интересном случае $d=10$ помещены в отдельный раздел.

\section{ПРИЛОЖЕНИЕ}

Формулы для $d \mathcal{P}_{d \mid s}^{\mu}$ при $d=4,6,8$ :

$$
\begin{aligned}
& \frac{d \mathcal{P}_{4 \mid s}^{\mu}}{d \tau}=\frac{4-12 s}{3} \pi \dot{u}^{2} u^{\mu}, \\
& \frac{d \mathcal{P}_{6 \mid s}^{\mu}}{d \tau}=\pi^{2}\left[\frac{19}{3}-(2 s-3)^{2}\right] \dot{u}^{4} u^{\mu}+\frac{8 \pi^{2}}{15}(1-5 s) \ddot{u}^{2} u^{\mu}+ \\
& +\frac{16 \pi^{2}}{35}(2-7 s)(\dot{u} \ddot{u}) \dot{u}^{\mu}+\frac{16 \pi^{2}}{105}(7 s-4) \dot{u}^{2} \ddot{u}^{\mu}, \\
& \frac{d \mathcal{P}_{8 \mid s}^{\mu}}{d \tau}=\left\{-\frac{16 \pi^{3}}{9}\left(\frac{7 s^{3}}{10}-\frac{135}{14} s^{2}+\frac{838}{35} s-5\right) \dot{u}^{6}+\frac{32 \pi^{3}}{3}\left(-s^{2}+\frac{101}{35} s-\frac{41}{63}\right)(\dot{u} \ddot{u})^{2}+\right. \\
& +\frac{16 \pi^{3}}{3}\left(-\frac{s^{2}}{5}+\frac{59}{35} s-\frac{20}{63}\right) \dot{u}^{2} \ddot{u}^{2}+32 \pi^{3}\left(\frac{s^{2}}{15}-\frac{2 s}{7}+\frac{11}{189}\right) \dot{u}^{2}(\dot{u} \dot{\ddot{u}})- \\
& \left.-\frac{16 \pi^{3}}{15}\left(s-\frac{1}{7}\right) \dot{\ddot{u}}^{2}\right\} u^{\mu}+ \\
& +\left\{\frac{32 \pi^{3}}{7}\left(-\frac{6 s^{2}}{5}+\frac{19}{5} s-\frac{283}{297}\right) \dot{u}^{2}(\dot{u} \ddot{u})+\frac{64 \pi^{3}}{7}\left(\frac{1}{27}-\frac{s}{5}\right) \ddot{u} \dot{\ddot{u}}\right\} \dot{u}^{\mu}+ \\
& +\left\{\frac{64 \pi^{3}}{21}\left(\frac{2 s^{2}}{5}-2 s+\frac{65}{99}\right) \dot{u}^{4}+\frac{64 \pi^{3}}{21}\left(\frac{1}{9}-\frac{2 s}{5}\right) \dot{u} \dot{u}\right\} \ddot{u}^{\mu}+ \\
& +\frac{32 \pi^{3}}{35}\left(s-\frac{17}{27}\right)(\dot{u} \ddot{u}) \dot{\ddot{u}}^{\mu} \text {. }
\end{aligned}
$$

Формулы для $P_{0}^{(d \mid s)}$ при $d=4,6,8$ :

$$
P_{0}^{(4 \mid s)}=\frac{12 s-4}{3} \pi \kappa_{1}^{2},
$$

1)Программу расчета можно найти, например, по адресу http://thesaurus.itep.ru/ project/0703mm/intens9.zip. 


$$
\begin{aligned}
P_{0}^{(6 \mid s)}= & -\pi^{2}\left(4 s^{2}-\frac{28}{3} s+\frac{32}{15}\right) 4 \kappa_{1}^{4}+\frac{8 \pi^{2}}{15}(5 s-1) \kappa_{1}^{2} \kappa_{2}^{2}, \\
P_{0}^{(8 \mid s)}= & \frac{16 \pi^{3}}{105}(7 s-1)\left(\kappa_{1}^{2} \kappa_{2}^{2} \kappa_{3}^{2}+\kappa_{1}^{2} \kappa_{2}^{4}\right)+ \\
& +\frac{8 \pi^{3}}{315}\left(49 s^{3}-549 s^{2}+1004 s-216\right) \kappa_{1}^{6}-16 \pi^{3}\left(\frac{s^{2}}{5}-s+\frac{64}{315}\right) \kappa_{1}^{4} \kappa_{2}^{2} .
\end{aligned}
$$

Формулы для $I_{d \mid s}$ при $d=4,6,8$ :

$$
\begin{aligned}
I_{4 \mid s}= & \frac{12 s-4}{3} \pi \kappa_{1}^{2} \\
I_{6 \mid s}= & -\pi^{2}\left(4 s^{2}-\frac{124}{15} s+\frac{32}{21}\right) \kappa_{1}^{4}+\frac{8 \pi^{2}}{15}(5 s-1)\left(\dot{\kappa}_{1}^{2}+\kappa_{1}^{2} \kappa_{2}^{2}\right) \\
I_{8 \mid s}= & \frac{16 \pi^{3}}{105}\left[\left(\frac{49}{6} s^{3}-\frac{167}{2} s^{2}+\frac{406}{3} s-\frac{276}{11}\right) \kappa_{1}^{6}-\right. \\
& \quad\left(21 s^{2}-97 s+\frac{172}{9}\right) \kappa_{1}^{4} \kappa_{2}^{2}-\left(77 s^{2}-180 s+\frac{109}{3}\right) \kappa_{1}^{2} \dot{\kappa}_{1}^{2}+ \\
& \left.+2\left(7 s^{2}-9 s+4\right) \kappa_{1}^{3} \ddot{\kappa}_{1}+(7 s-1)\left\{\kappa_{1}^{2} \kappa_{2}^{2} \kappa_{3}^{2}+\left(\kappa_{1} \kappa_{2}^{2}-\ddot{\kappa}_{1}\right)^{2}+\left(2 \kappa_{2} \dot{\kappa}_{1}+\kappa_{1} \dot{\kappa}_{2}\right)^{2}\right\}\right] .
\end{aligned}
$$

Результаты для наиболее интересного случая $d=10$ :

$$
\begin{aligned}
\frac{d \mathcal{P}_{10 \mid s}^{\mu}}{d \tau}= & \left\{\frac{4 \pi^{4}}{315}\left(19 s^{4}-\frac{6466}{9} s^{3}+\frac{236237}{33} s^{2}-\frac{1609666}{99} s+\frac{9800}{3}\right) \dot{u}^{8}-\right. \\
& -\frac{32 \pi^{4}}{7}\left(s^{3}-\frac{200}{9} s^{2}+\frac{2147}{33} s-\frac{6230}{429}\right) \dot{u}^{2}(\dot{u} \ddot{u})^{2}+ \\
& +\frac{16 \pi^{4}}{35}\left(s^{3}-\frac{61}{27} s^{2}-\frac{8316}{297} s+\frac{11690}{1287}\right) \dot{u}^{4} \ddot{u}^{2}+ \\
& +\frac{64 \pi^{4}}{35}\left(s^{3}-\frac{1037}{54} s^{2}+\frac{32653}{594} s-\frac{4865}{429}\right) \dot{u}^{4}(\dot{u} \dot{u})- \\
& -\frac{16 \pi^{4}}{105}\left(19 s^{2}-\frac{611}{9} s+\frac{512}{33}\right) \ddot{u}^{4}-\frac{32 \pi^{4}}{15}\left(\frac{17}{7} s^{2}-151 s+\frac{34}{21}\right)(\dot{u} \dot{u})^{2}- \\
& -\frac{64 \pi^{4}}{105}\left(s^{2}-\frac{53}{3} s+\frac{100}{33}\right)(\dot{u} \ddot{u})(\ddot{u} \dot{\ddot{u}})-\frac{256 \pi^{4}}{35}\left(s^{2}-\frac{263}{108} s+\frac{119}{198}\right) \ddot{u}^{2}(\dot{u} \dot{u})- \\
& -\frac{32 \pi^{4}}{105}\left(s^{2}-\frac{134}{9} s+\frac{25}{11}\right) \dot{u}^{2} \dot{\ddot{u}}{ }^{2}+\frac{64 \pi^{4}}{105}\left(s^{2}-\frac{74}{9} s+\frac{15}{11}\right) \dot{u}^{2}(\ddot{u} \ddot{u})+ \\
& \left.+\frac{64 \pi^{4}}{315}\left(9 s^{2}-49 s+10\right)(\dot{u} \ddot{u})(\dot{u} \ddot{u})+\frac{32 \pi^{4}}{105}\left(\frac{1}{9}-s\right) \ddot{u}^{4}\right\} u^{\mu}- \\
& -\left\{\frac{32 \pi^{4}}{63}\left(\frac{8 s^{3}}{3}-\frac{593}{11} s^{2}+\frac{72173}{429} s-\frac{5670}{143}\right) \dot{u}^{4}(\dot{u} \ddot{u})+\right. \\
& +\frac{64 \pi^{4}}{189}\left(20 s^{2}-\frac{829}{11} s+\frac{2664}{143}\right) \ddot{u}{ }^{2}(\dot{u} \ddot{u})+ \\
& \left.+5 s^{2}-\frac{133}{11} s+\frac{453}{143}\right)(\dot{u} \dot{u})(\dot{u} \ddot{u})-\frac{128 \pi^{4}}{189}\left(s^{2}-\frac{109}{22} s+\frac{147}{143}\right) \dot{u}^{2}(\dot{u} \ddot{u})+
\end{aligned}
$$




$$
\begin{aligned}
& \left.+\frac{320 \pi^{4}}{819}\left(1-\frac{208}{33} s\right) \dot{u}^{2}(\ddot{u} \dot{u})+\frac{64 \pi^{4}}{189}\left(2 s-\frac{3}{11}\right)(\dot{\ddot{u}} \ddot{u})\right\} \dot{u}^{\mu}+ \\
& +\left\{\frac{128 \pi^{4}}{189}\left(s^{3}-\frac{785}{44} s^{2}+\frac{30843}{572} s-\frac{2065}{143}\right) \dot{u}^{6}+\right. \\
& +\frac{320 \pi^{4}}{189}\left(s^{2}-\frac{53 s}{11}+\frac{216}{143}\right)(\dot{u} \ddot{u})^{2}-\frac{640 \pi^{4}}{189}\left(s^{2}-4 s+\frac{1569}{1430}\right) \dot{u}^{2}(\dot{u} \dot{\ddot{u}})- \\
& \left.-\frac{64 \pi^{4}}{189}\left(4 s^{2}+s+\frac{123}{143}\right) \dot{u}^{2} \ddot{u}^{2}+\frac{128 \pi^{4}}{189}\left(\frac{2}{11}-s\right)(\ddot{u} \ddot{u})\right\} \ddot{u}^{\mu}+ \\
& +\left\{\frac{256 \pi^{4}}{4158}\left(22 s^{2}-167 s+\frac{795}{13}\right) \dot{u}^{2}(\dot{u} \ddot{u})+\frac{64 \pi^{4}}{189}\left(\frac{3}{11}-s\right)(\dot{u} \ddot{u})\right\} \dot{\ddot{u}}^{\mu}- \\
& -\left\{\frac{32 \pi^{4}}{945}\left(s^{2}-\frac{141}{11} s+\frac{1020}{143}\right) \dot{u}^{4}-\frac{64 \pi^{4}}{315}\left(s-\frac{23}{33}\right) \ddot{u}^{2}-\right. \\
& \left.-\frac{256 \pi^{4}}{945}\left(s-\frac{29}{44}\right)(\dot{u} \dot{u})\right\} \ddot{u}^{\mu} \text {; } \\
& P_{0}^{(10 \mid s)}=\frac{32 \pi^{4}}{315}\left[\left(-\frac{19}{8} s^{4}+\frac{2747}{36} s^{3}-\frac{152021}{264} s^{2}+\frac{369827}{396}-192\right) \kappa_{1}^{8}+\right. \\
& +\left(\frac{9}{2} s^{3}-605 s^{2}+933 s-188\right) \kappa_{1}^{6} \kappa_{2}^{2}- \\
& -\left(\frac{33}{2} s^{2}-325 s+28\right) \kappa_{1}^{4} \kappa_{2}^{4}-\left(9 s^{2}-\frac{88}{3} s+\frac{43}{3}\right) \kappa_{1}^{4} \kappa_{2}^{2} \kappa_{3}^{2}+ \\
& \left.+\left(3 s-\frac{1}{3}\right)\left(\kappa_{1}^{2} \kappa_{2}^{6}+\kappa_{1}^{2} \kappa_{2}^{2} \kappa_{3}^{2} \kappa_{4}^{2}+2 \kappa_{1}^{2} \kappa_{2}^{4} \kappa_{3}^{2}+\kappa_{1}^{2} \kappa_{2}^{2} \kappa_{3}^{4}\right)\right] \\
& I_{10 \mid s}=\frac{16 \pi^{4}}{945}\left[\left(81 s^{3}-1693 s^{2}+\frac{53244}{11} s-\frac{133048}{143}\right) \kappa_{1}^{6} \kappa_{2}^{2}+\right. \\
& +\left(243 s^{3}-4785 s^{2}+\frac{106818}{11} s-\frac{300280}{143}\right) \kappa_{1}^{4} \dot{\kappa}_{1}^{2}- \\
& -\left(99 s^{2}-931 s+\frac{1744}{11}\right) \kappa_{1}^{4} \kappa_{2}^{4}+\left(36 s^{2}-220 s+\frac{416}{11}\right) \kappa_{1}^{4} \kappa_{2} \ddot{\kappa}_{2}+ \\
& +\left(324 s^{2}-1780 s+\frac{3392}{11}\right) \kappa_{1}^{3} \ddot{\kappa}_{1} \kappa_{2}^{2}-\left(468 s^{2}-1160 s+\frac{2812}{11}\right) \kappa_{1} \ddot{\kappa}_{1} \dot{\kappa}_{1}^{2}+ \\
& +\left(144 s^{2}-568 s+\frac{1336}{11}\right) \kappa_{1}^{2} \dot{\kappa}_{1} \dot{\kappa}_{1}-\left(171 s^{2}-413 s+\frac{1062}{11}\right) \dot{\kappa}_{1}^{4}- \\
& -\left(54 s^{2}-488 s+\frac{866}{11}\right) \kappa_{1}^{4} \kappa_{2}^{2} \kappa_{3}^{2}- \\
& -\left(\frac{57}{4} s^{4}-\frac{2507}{6} s^{3}+\frac{125989}{44} s^{2}-\frac{3644975}{858} s+\frac{111744}{143}\right) \kappa_{1}^{8}- \\
& -\left(108 s^{3}-1506 s^{2}+\frac{31794}{11} s-\frac{83808}{143}\right) \kappa_{1}^{5} \ddot{\kappa}_{1}- \\
& -\left(324 s^{2}-822 s+\frac{1878}{11}\right) \kappa_{1}^{2} \ddot{\kappa}_{1}^{2}-\left(432 s^{2}-2752 s+\frac{5760}{11}\right) \kappa_{1}^{3} \dot{\kappa}_{1} \kappa_{2} \dot{\kappa}_{2}-
\end{aligned}
$$




$$
\begin{aligned}
& -\left(18 s^{2}-268 s+\frac{450}{11}\right) \kappa_{1}^{4} \dot{\kappa}_{2}^{2}-\left(450 s^{2}-3714 s+\frac{7520}{11}\right) \kappa_{1}^{2} \dot{\kappa}_{1}^{2} \kappa_{2}^{2}+ \\
& +2(9 s-1)\left[\left(\dot{\kappa}_{1}-3 \dot{\kappa}_{1} \kappa_{2}^{2}-3 \kappa_{1} \kappa_{2} \dot{\kappa}_{2}\right)^{2}+\right. \\
& +\left(3 \ddot{\kappa}_{1} \kappa_{2}+3 \dot{\kappa}_{1} \dot{\kappa}_{2}+\kappa_{1} \ddot{\kappa}_{2}-\kappa_{1} \kappa_{2}^{3}\right)^{2}+\left(2 \kappa_{1} \dot{\kappa}_{2} \kappa_{3}+\kappa_{1} \kappa_{2} \dot{\kappa}_{3}+3 \dot{\kappa}_{1} \kappa_{2} \kappa_{3}\right)^{2}- \\
& \left.\left.-\kappa_{1}^{2} \kappa_{2}^{2} \kappa_{3}^{2}\left(2 \kappa_{2}^{2}+\kappa_{3}^{2}+\kappa_{4}^{2}\right)-2 \kappa_{1} \kappa_{2} \kappa_{3}^{2}\left(3 \ddot{\kappa}_{1} \kappa_{2}+\kappa_{1} \ddot{\kappa}_{2}+3 \dot{\kappa}_{1} \dot{\kappa}_{2}\right)\right]\right] .
\end{aligned}
$$

Заметим, что в выражениях для излученного импульса коэффициенты перед членами $k$-й степени по $U_{i j}$ являются полиномами степени $k$ от ранга $s$. Относительная простота коэффициентов этих полиномов подразумевает, что для них может существовать общая формула в произвольной размерности $d$. Однако хотелось бы снова подчеркнуть, что использование всех этих выражений для излучения полей старших рангов ограниченно из-за необходимости учета вкладов других тензоров энергии-импульса. Кроме того, чтобы отделить вклад поля спина s от вклада поля ранга $s$, нужно добавить подходящую комбинацию тензоров энергии-импульса полей низших рангов. Это можно сделать непосредственно, если использовать формулы, полученных в данном тексте.

Благодарности. Мы благодарны П. Казинскому и С. Ляховичу за информативные обсуждения. Данная работа частично поддержана программой Государственного агенства по атомной энергии, РФФИ (гранты № 07-02-00878-а (А. Д. Миронов) и № 07-02-00645 (А. Ю. Морозов), совместный грант № 06-01-92059-КЭ_а), Программой поддержки ведущих научных школ (грант № НШ-8004.2006.2), проектом NWO 047.011.2004.026, ИНТАС (грант № 05-1000008-7865) и проектом ANR-05-BLAN0029-01.

\section{Список литературы}

[1] Л. Д. Ландау, Е. М. Лифшиц, Теоретическая физика. Т. ІІ. Теория поля, Физматлит, М., 2001; Дж. Джексон, Классическал электродинамика, Мир, М., 1965; А. А. Соколов, И. М. Тернов, В. Ч. Жуковский, А. Б. Борисов, Квантовая электродинамика, Изд-во МГУ, 1983; С. Вайнберг, Гравитачия и космология, Мир, М., 1975; И. Хриплович, Общая теория относительности, РХД, Ижевск, 2001; В. Kosyakov, Introduction to the Classical Theory of Particles and Fields, Springer, Berlin, 2007.

[2] N. Arkani-Hamed, S. Dimopoulos, G. Dvali, Phys. Lett. B, 429:3-4 (1998), 263-272; Phys. Rev. D, 59:8 (1999), 086004; I. Antoniadis, N. Arkani-Hamed, S. Dimopoulos, G. Dvali, Phys. Lett. B, 436:3-4 (1998), 257-263.

[3] T. Banks, W. Fischler, A model for high energy scattering in quantum gravity, arXiv: hep-th/9906038; S. B. Giddings, S. Thomas, Phys. Rev. D, 65:5 (2002), 056010; S. Dimopoulos, G. Landsberg, Phys. Rev. Lett., 87:16 (2001), 161602; P. Kanti, Internat. J. Modern Phys. A, 19:29 (2004), 4899-4951; G. Landsberg, J. Phys. G, 32:9 (2006), R337-365; arXiv: hep-ph/0607297; M. Cavaglia, R. Godang, L. Cremaldi, D. Summers, Comput. Phys. Comm., 117:6 (2007), 506-517; arXiv: hep-ph/0609001; A. Mironov, A. Morozov, T. Tomaras, Can Centauros or Chirons be the first observations of evaporating mini black holes?, arXiv: hep-ph/0311318; A. Cafarella, C. Corianò, T. Tomaras, JHEP, 06 (2005), 065; arXiv: hep-ph/0410358; L. Anchordoqui, T. Paul, S. Reucroft, J. Swain, Internat. J. Modern Phys. A, 18:13 (2003), 2229-2366; A. Mironov, A. Morozov, T. Tomaras, Facta Univ. Ser. Phys. Chem. Tech., 4 (2006), 381-404; arXiv: 0710. 3395. 
[4] Б. П. Косяков, ТМФ, 119:1 (1999), 119-135; arXiv: hep-th/0207217.

[5] D. Galt'sov, Phys. Rev. D, 66:2 (2002), 025016; arXiv: hep-th/0112110; P. O. Kazinski, S.L. Lyakhovich, A. A. Sharapov, Phys. Rev. D, 66:2 (2002), 025017; arXiv: hep-th/0201046; V. Cardoso, O. Dias, J. Lemos, Phys. Rev. D, 67:6 (2003), 064026; arXiv: hep-th/0212168; Yu. Yaremko, J. Phys. A, 37:3 (2004), 1079-1091; B. Koch, M. Bleicher, Писъма в ЖКЭТФ, 87:2 (2008), 75-80; arXiv: hep-th/0512353; P. Krtouš, J. Podolský, Classical Quantum Gravity, 23:5 (2006), 1603-1615; arXiv: gr-qc/0602007; H. A. Morales-Técotl, O. Pedraza, L. O. Pimentel, Low-energy effects in brane worlds: Liennard-Wiechert potentials and Hydrogen Lamb shifteprint, arXiv: physics/0611241.

[6] M. Gürses, O. Sarıŏlu, Classical Quantum Gravity, 19:16 (2002), 4249-4261; 20:2 (2003), 351-358; J. Math. Phys., 44:10 (2003), 4672-4680; arXiv: hep-th/0303078.

[7] V. Cardoso, M. Cavaglia, J.-Q. Guo, Gravitational Larmor formula in higher dimensions, arXiv: hep-th/0702138.

[8] А. Миронов, А. Морозов, Писвма в ЖЭТФ, 85:1 (2007), 9-14; arXiv: hep-ph/0612074.

[9] M. Spivak, A Comprehensive Introduction to Differential Geometry, V. I, Publish or Perish, Berkeley, California, 1979. 